\title{
Science with radio pulsar astrometry
}

\section{Shami Chatterjee}

Cornell University, United States

email: shami@astro.cornell.edu

\begin{abstract}
High precision astrometry on radio pulsars can provide model-independent estimates of their distances and velocities. Such estimates serve to calibrate models of the Galactic electron density distribution, thereby improving distance estimates for the entire pulsar population. They can provide independent astrometric information for precision pulse timing, reducing the number of fit parameters and thus potentially improving the sensitivity of pulsar timing arrays to the gravitational wave background. Individual neutron stars also serve as laboratories for astrophysics. For example, distances to highly luminous recycled pulsars identified by the Fermi gamma ray space telescope will constrain their energetics and may serve to probe the equation of state for nuclear matter at extremes of density and pressure. Here we provide an update on ongoing astrometry programs with the Very Long Baseline Array and the scientific results from these efforts.
\end{abstract}

